\title{
Heart Rate Regulation During Cycle-Ergometer Exercise via Event-Driven Biofeedback
}

\author{
Ahmadreza Argha · Steven W. Su · Branko G. Celler
}

the date of receipt and acceptance should be inserted later

\begin{abstract}
This paper is devoted to the problem of regulating the heart rate (HR) response along a predetermined reference profile, for cycle-ergometer exercises designed for training or cardio-respiratory rehabilitation. The controller designed in this study is a non-conventional, non-modelbased, proportional, integral and derivative (PID) controller. The PID controller commands can be transmitted as biofeedback auditory commands, which can be heard and interpreted by the exercising subject to increase or reduce exercise intensity. However, in such a case, for the purposes of effectively communicating to the exercising subject a change in the required exercise intensity, the timing of this feedback signal relative to the position of the pedals becomes critical. A feedback signal delivered when the pedals are not in a suitable position to efficiently exert force may be ineffective and this may, in turn, lead to the cognitive disengagement of the user from the feedback controller. This note examines a novel form of control system which has been expressly designed for this project. The system is called an "actuatorbased event-driven control system". The proposed control system was experimentally verified using twenty four healthy male subjects who were randomly divided into two separate groups, along with cross validation scheme. A statistical analysis was employed to test the generalisation of the PID tunes, derived based on the average transfer functions of the two groups, and it revealed that there were no significant differences between the mean values of root-meansquare of the tracking error of two groups [3.9 vs. $3.7 \mathrm{bpm}$,
\end{abstract}

A. Argha and S. W. Su

are with the Faculty of Engineering and Information Technology, University of Technology, Sydney, PO Box 123, Broadway, NSW 2007, Australia.

B. G. Celler

is currently a Research Professor at the University of New South Wales, School of Electrical Engineering, Sydney, Australia. E-mail: b.celler@unsw.edu.au $p=0.65]$. Furthermore, the results of a second statistical hypothesis test showed that the proposed PID controller with novel synchronised biofeedback mechanism has better performance compared to a conventional PID controller with a fixed-rate biofeedback mechanism [Group 1: 3.9 vs. 5.0 bpm, Group 2: 3.7 vs. 4.4 bpm, $p<0.05]$.

Keywords Heart rate regulation · actuator-based eventdriven PID control · cycle-ergometer exercising

\section{Introduction}

It is generally accepted that regular aerobic exercise has a positive effect on reducing the risk of heart disease, rehabilitation post infarct and for reversing the health effects of diabetes or overweight $[5,25,1,16]$.

Heart rate (HR) can be used as an index to monitor the exercise intensity [18]. This fact makes it simpler to develop a control system to steer the human HR to a predetermined and individual exercise prescription, represented as a target HR profile, instead of directly employing the exercise intensity in kJoules or Watts and exercise rate (ER) as control parameters.

Automated exercise testing systems have become more and more important in several areas such as sport training, medical diagnosis, rehabilitation and analysis of cardiorespiratory kinetics $[6,23]$. Moreover, controlling the HR during exercise is of great importance in exercise protocols designed for patients with cardiovascular diseases and those recovering from an infarct or cardiac surgery $[4,13]$.

Cycle-ergometers are often considered preferable to treadmills, in terms of cost, floor space occupied, level of noise pollution and the risk of falling or injury. Moreover, the upper body motion is usually reduced during cycle-ergometer exercises, compared to the treadmill exercises, and hence, 


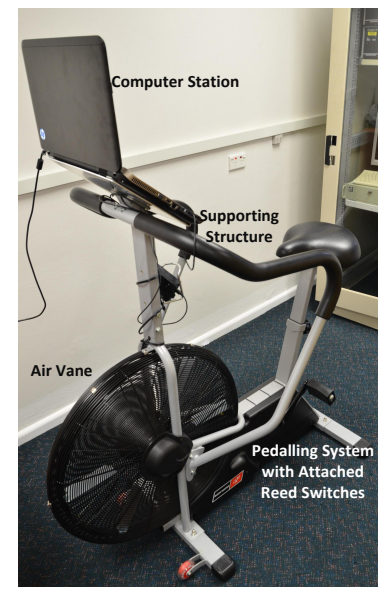

Fig. 1.1 Cycle-ergometer exercising system

measuring the HR is easier and more reliable [8]. Cycleergometers with an externally controllable automatic braking system has been utilised in the literature as an automated exercise system whose mechanism works based on adjusting the resistance of the brake rather than the pedalling rate to change the exercise level [14]. However, the cycleergometer with built-in braking system is often expensive and may not be suitable for use at home as a rehabilitation device. In this project, we have alternatively selected a vane bike that is low cost, and very sturdy. The design is also particularly safe and suitable for use by frail elderly patients. Moreover, the cooling effect of the air vane bike can be regarded as a distinct advantage, see Fig. 1.1. On the other hand, in such a case, the controller signal must be transmitted directly to the subject through some sort of biofeedback. Biofeedback has been used in rehabilitation [10] for more than fifty years. In general, it serves to provide biological information for patients in real-time and to motivate them to implement desired tasks [10]. Biofeedback usually requires the measurement of a subject's biomedical variables and transference to the subject through either direct feedback according to the measured variables, i.e. displaying a numerical value on a wearable device (e.g. watch), or converted feedback, i.e. a mechanism designed to generate an auditory signal, visual display or tactile feedback based on the subject's measured biomedical variables $[10,19,17]$.

Existing auditory (or visual) biofeedback mechanisms, e.g. in [3], are not appropriate for this project, as they may lead to a cognitive disengagement of the subject from the feedback controller and possible abrupt changes in the subject's HR reference. We have developed a different mechanism for generating and transmitting biofeedback that needs a novel control system strategy, which can smooth out the abrupt changes in the control signals preventing overshooting in the HR responses, and allowing HR to vary in a more

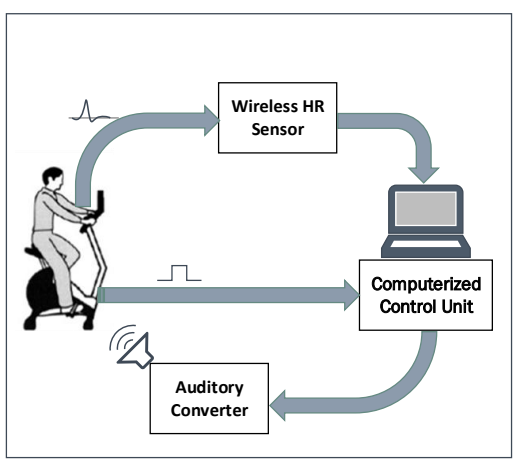

Fig. 1.2 Block diagram for the HR regulation system during cycling exercise

gradual and smoother manner. This smooth variation of HR response is a very important parameter in the automated exercise testing systems developed for the cardiac rehabilitation programs. The novel control system, regarded here as an actuator-based event-driven control system, is different to the existing sensor-based event-driven control system in the literature [12].

A number of sophisticated strategies have been utilised for real-time control of HR during exercises, e.g. $\mathrm{H}_{\infty}$ control $[4,24]$ and model predictive control [21,22]. However, owing to the simplicity of the proportional, integral and derivative (PID) control structure, it is usually preferable to other complex control strategies in practical applications. Moreover, it is well known that the HR response to dynamic exercise is nonlinear and it may be different for each exercising subject, in different physical situations [3]. Hence, an adaptive and model-based control method is usually designed to address the problem of HR control. When using PID control, the controller parameters for each subject should be determined continuously using a subject specific model. Alternatively, one can design a robust PID controller which is able to compensate for the inter-individual differences in the dynamic HR response to the work rate [14].

In this paper, an event-driven control system is proposed, in which two anti-windup [2] mechanisms are designed to protect the user against possible large HR fluctuations owing to inaccurate controller parameters and/or individual HR responses. This event-driven control system consists of a PID controller whose output signal is translated to a set of auditory stimulus transmitted to the exercising subject at a timevarying rate synchronised with the subject's pedalling rate and position of the pedals.

The novel control system was experimentally verified employing cross-validation scheme. In doing so, firstly, we tuned the proposed control system parameters using two obtained nominal average HR step response of two separate group of participants. Then the resulted controller parameters were validated with the aid of a different group of exercising subjects. Moreover, statistical analysis of the obtained 


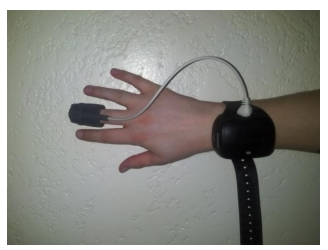

Fig. 2.1 Nonin 4100 Pulse Oximeter

average tracking error signals illustrated that the proposed actuator-based event-driven PID controller has better performance compared to a conventional PID controller with a fixed-rate biofeedback mechanism.

\section{Methods}

\subsection{Subjects}

Twenty four healthy male subjects (free from any known metabolic or cardiac disorders, hypertension, and not under any medication) participated in this project to exercise on the cycle-ergometer. Written informed consents were obtained from all participants, and the study was approved by the UTS Human Research Ethics Committee (UTS HREC 2009000227). Since several parameters, such as the time of the day, emotional state, temperature, humidity, previous activity, food and caffeine intake, fatigue, altitude and dehydration, can influence HR [15], the subjects were asked to have a light meal two hours before the experiment and not to get involved in intense exercise for 24 hours before the experiment. Environmental conditions were maintained constant for all subjects. The physical characteristics of the subjects are presented in Table 1 (values are: mean \pm standard deviation, range). As seen from Table 1, we divided the participants into two separate groups at random, each of them including twelve participants.

Table 1 Physical characteristics of participants

\begin{tabular}{ccc}
\hline & Group 1, n=12 & Group 2, n=12 \\
\hline Age $($ years $)$ & $27.7 \pm 5.0,21-36$ & $30.4 \pm 6.7,23-47$ \\
Height $(\mathrm{cm})$ & $176.2 \pm 4.7,169-184$ & $173.7 \pm 6.0,163-186$ \\
Weight $(\mathrm{Kg})$ & $71.2 \pm 13.3,55-95$ & $73.6 \pm 8.3,59-84$ \\
BMI $\left(\frac{\mathrm{Kg}}{\mathrm{m}^{2}}\right)$ & $22.6 \pm 3.7,18-28$ & $24.2 \pm 1.8,21-27$ \\
\hline
\end{tabular}

\subsection{Equipment and data acquisition system}

The Nonin 4100 bluetooth Pulse oximeter was selected for our project (see Fig. 2.1) as it can provide a number of useful outputs such as $\mathrm{SpO}_{2}$ (peripheral capillary oxygen saturation) and $\mathrm{HR}$ as well as the photo plethysmographic wave-

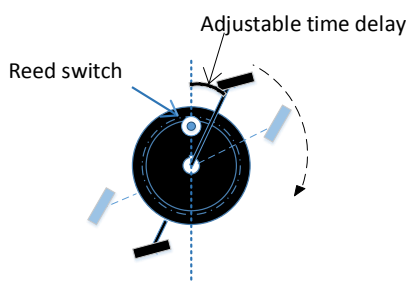

Fig. 2.2 Reed switch and adjustable time delay parameter

forms. Whilst numerous algorithms have been published [9] for the reliable detection of HR from the photo-plethysmographic waveform we have selected to use HR data provided by the Nonin device every second. Whilst this derivation could be subject to movement artefacts and other disturbances, our experiments setup makes it possible to stabilise the hand on the handlebar of the air bike to minimise these artefacts and to maximise blood flow to the finger. We have exhaustively compared the HR output from the Nonin oximeter against the Polar ECG strap and against direct recordings of a single channel ECG, and in our experimental setup the results for HR were almost identical. Moreover, the cycleergometer that we used in this project is an air vane exercise bike, which is well suited for safe exercise by the frail and/or elderly. Since it has a big supporting structure, which can be used by the exercising patient to stabilise themselves during exercising, the finger pulse oximeter is almost completely stable during exercise and it generates minimal artefact. We have developed a data acquisition system using $\mathrm{Na}$ tional Instrument LabVIEW which provides easy synchronisation and a graphical user interface. The LabVIEW collected HR signal from the Nonin 4100 pulse oximeter every $1 s$. In addition, to prepare a reliable real-time HR signal for the computer controller, an exponentially weighed moving average filter (see [7]), with filter coefficient $\alpha=0.75$, is implemented.

Two reed switches and magnets have been attached to the crank shaft of the pedals of the exercise bike, in order to provide a pulse whenever a full revolution has been completed; see Fig. 2.2. In the Labview program, a time-delay parameter may be set to help the user to adjust the timedelay between the position of the pedal sensed by the sensor and the time when the command is sent. This parameter is useful since we can adjust the point where the user can most effectively apply force to the pedals; see Fig. 2.2.

\subsection{HR profile}

The HR profile in this work is selected in three stages. In the first stage, called the warm up period, we aim to gradually increase the user HR from their normal HR $\left(H R_{n}\right)$ to the exercise $\mathrm{HR}\left(H R_{e}\right)$ which then remains constant for a while 
$\left(T_{e}\right)$. The warm up slope is

$\theta_{w}=\frac{H R_{e}-H R_{n}}{T_{w}}$,

in which $\theta_{w}$ represents the warm up slope and $T_{w}$ shows the warm up time duration. The warm up time duration $T_{w}$ is used to avoid an excessively rapid increase in the HR. The second aerobic training stage is denoted by $T_{e}$, which may be computed according to the maximum HR. For obtaining the ideal target aerobic training HR, various formulas have been proposed. The maximum HR $\left(H R_{\text {max }}\right)$ is commonly characterised by the well-known Haskell and Fox formula [20] as:

$H R_{\max }=220-$ Age

We then set the exercising $H R_{e}$ as $0.65 \sim 0.85$ of $H R_{\text {max }}$, depending on the subject's level of fitness and/or risk profile. The application of the third cooling down or recovery stage $\left(T_{c}\right)$ is basically to prevent possible venous pooling and reduced venous return to the heart from an excessively abrupt termination of the exercise.

Remark 1 In this project, the HR profile is dynamic. In other words, the HR profile always starts from the subject's current resting $\mathrm{HR}$ and increases to a value which is calculated based on the age of the subject (aerobic training ideal HR).

\subsection{Control System}

Most commonly, control theory and engineering consider control systems to be time-driven where continuous-time signals are expressed by their sampled values at a fixed sample frequency. This means that the sampling intervals are assumed to be fixed, hence, the analysis problems can be implemented by utilising the existing literature on the sampled data systems. The control actions are also assumed to be generated at a frequency equal to the sampling frequency, which is referred to as synchronous in time, or time-driven control.

However, for event-driven controllers [12], the occurrence of a certain event triggers the controllers actions. Also, the sampling rate of the HR transmitted to the program by an ECG or pulse oximeter transducer is typically constant (1 sample per second), however, the rate of sending the commands to the exercising subject varies according to the pedalling rate. This form of control system, called here an actuator based event-driven control system, is obviously different from the existing control systems in [12] for the sensor-based event-driven control systems. This is because the aim of this control system is to control the exercise rate through the heart rate and not to control the velocity or the position of a rotary system (DC motor in the aforementioned control systems and the pedalling system here) and the HR sensor signal, sampled with a constant rate independent from the pedalling rate, will not trigger the control actions. Fig. 2.3 gives a general view of the asynchronous signals in the designed control system.

Now three possible methods can be considered, which are as follows:

1) Updating the control signal for each event using the latest available information. However, owing to the existence of the integral action in the control law, this control strategy may be more likely to suffer from windup phenomena in the system performance.

2) Designing a control system which utilises an observerbased framework to estimate firstly the next actuator event instant (the instant that the left or right pedal passes the reed sensor) and, secondly, the HR value at asynchronous controller event instants, using synchronous measurement instants. Although this observer-based method may have a better performance compared to the conventional control schemes, it has a major drawback, which is the intensive computational burden on the processor, as it needs to deal with a time-varying observer-based control system.

3) Adopting a time-driven PID controller in the velocity form to our problem as follows.

Consider a time-driven PID controller in the velocity form such as

$$
u(k)=u(k-1)+\Delta u(k)
$$

where

$$
\begin{aligned}
\Delta u(k)= & K_{p}[e(k)-e(k-1)]+K_{i} T_{s} e(k) \\
& +\frac{K_{d}}{T_{s}}[e(k)-2 e(k-1)+e(k-2)],
\end{aligned}
$$

in which

$e(t)=r(t)-y(t)$

where $u(t), r(t), y(t), K_{p}, K_{i}$ and $K_{d}$ are control effort, reference, actual output, proportional gain, integral gain and derivative gain, respectively, and $T_{S}$ is a fixed sampling rate and is equivalent to the sampling period of the HR sensor. Then, whenever an event occurs (the left or right pedal passes the reed sensor), the last prepared update of the control signal will be transmitted as a biofeedback signal. As seen, the sampling rate of the HR transmitted to the program by the HR transducer, and thus, the updating rate of the control signal in (3), are typically constant (1 sample per second). However, the rate of biofeedback transmission to the exercising subject varies based on the pedalling rate. An immediate advantage of the last method is that it can assist in preventing the converted control commands (auditory stimulus) from reaching their limitations (see Subsections 2.4.1 and 2.4.3) and the system working under a saturation situation.

It is also well known that the heart rate response to exercise can be approximated as a first order system, where the time 

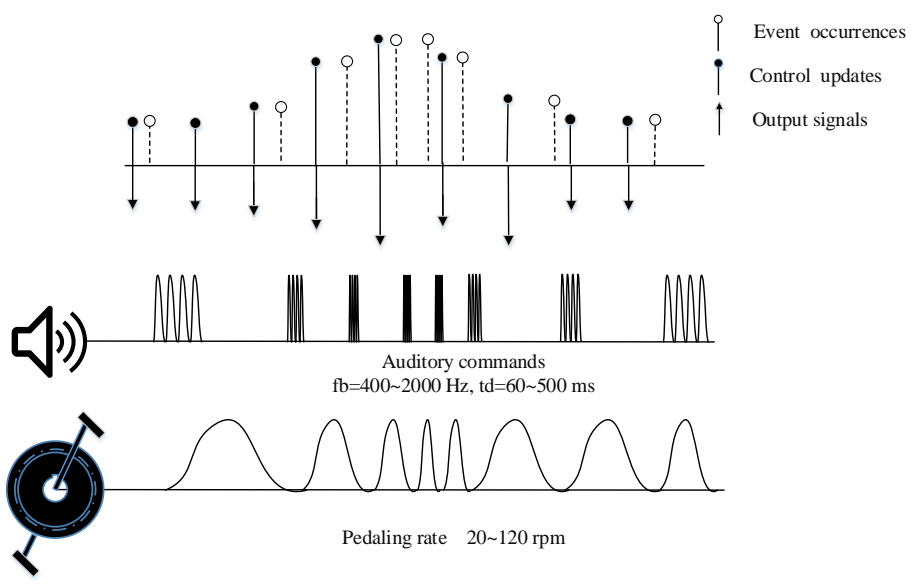

Fig. 2.3 Mechanism of the proposed control system.

constant is relatively slow [11]. Thus, the variation of the human HR is usually limited in a sampling period (say $1 s$ ). So, even if the controller uses the latest available sensor's information, rather than the actual one, the obtained control signal will be close to the desired one. Moreover, the number of additional events in the sampling period of the HR sensor is not big. With this specific bicycle, we found that the pedalling rate varies in the range $20 \sim 120 \mathrm{rpm}$, which means that at most, 2 or 3 events may occur in the fixed sampling period. Basically, the additional auditory signals are used here to instruct the subject to retain the proper exercise rate. Although the proposed control scheme here is very simple, our experimental studies (given in this paper) prove its effectiveness. As opposed to this, the lack of auditory commands for additional events occurring during the HR sampling time, make for an uncomfortable exercising experience for the subject.

\subsubsection{Auditory convertor}

In order to instruct the user to apply more or less effort at the right time, a combination of varying both the time duration and the frequency of auditory stimulus was used in this project. In this method a shorter time duration and lower frequency is used to motivate the subject to reduce the application of force to the pedals and conversely to increase the applied force if the time duration and frequency increases. However, the time intervals and frequency range which can be used are limited. We know that the time durations of more than a specific value result in the so-called aliasing phenomena. Additionally, time durations which are less than a specific value may not be heard by the subjects. This issue also holds for the frequency of the auditory signal. Accordingly, we selected the following range of time duration and frequency for the auditory stimulus: $60 \sim 500 \mathrm{~ms}$ and $400 \sim 2000 \mathrm{~Hz}$, respectively. The auditory converter thereby

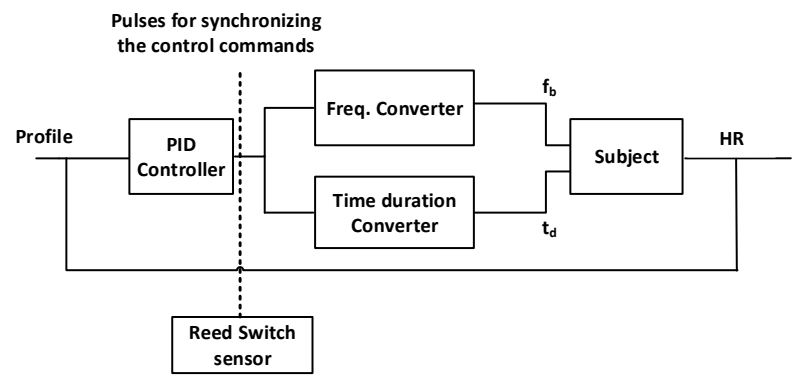

Fig. 2.4 The control system block diagram.

contains two saturation functions,

$t_{d}=\operatorname{sat}\{g(u(k))\}=\left\{\begin{array}{lr}\lambda_{1} & g(u(k)) \leq \lambda_{1} \\ g(u(k)) & \lambda_{1}<g(u(k))<\lambda_{2} \\ \lambda_{2} & g(u(k)) \geq \lambda_{2},\end{array}\right.$

where $t_{d}$ is the time duration of the auditory signals and $g(u(k))$ is a scaling function which converts the range of $u(k)$ to the range of the time duration, $\lambda_{1}=60 \mathrm{~ms}, \lambda_{2}=$ $500 \mathrm{~ms}$, and

$f_{b}=\operatorname{sat}\{h(u(k))\}=\left\{\begin{array}{lr}\gamma_{1} & h(u(k)) \leq \gamma_{1} \\ h(u(k)) & \gamma_{1}<h(u(k))<\gamma_{2} \\ \gamma_{2} & h(u(k)) \geq \gamma_{2},\end{array}\right.$

where $f_{b}$ is the frequency of the auditory signals and $h(u(k))$ is a scaling function which converts the range of $u(k)$ to the range of appropriate frequencies of the auditory signal, $\gamma_{1}=400 \mathrm{~Hz}$ and $\gamma_{2}=2000 \mathrm{~Hz}$.

Remark 2 It should be noted that the above system includes two distinct control loops. In simple terms, the control system is a MISO (2I1O) in which the PID controller of both control loops have the same parameters; see Fig. 2.4. 


\subsubsection{The proposed control mechanism in summary}

The structure of the designed control system can be briefly expressed as follows:

1- The control signals are updated synchronously with the HR measurements at a constant rate.

2- An auditory signal (biofeedback) is generated according to the level of the control signal and transmitted whenever the first event occurs.

3- This auditory signal motivates the exercising subject to increase or decrease their pedalling rate and the level of force applied.

4- As a result of varying the pedalling rate during some sampling periods, more than one event can occur. For these events, the same auditory signal as the one transmitted at the first event is sent to the subject. These additional auditory commands help the subject to continue tracking the desired profile.

Fig. 2.3 describes schematically the control system designed for the task.

\subsubsection{Two novel anti-windup mechanisms}

In the case of utilising an integrator in the controller, if the actuators perform at their limits independently of the system outputs measured, the error will be integrated continuously for a long period before conditions return to normal. Hence, the control effort may increase to a very large value. Thus the PID controller may cause large transients while the system actuator saturates. This is referred to as windup phenomena [2]. In this work, as the exercising subject plays the role of system actuator and has exercise limitations, windup can similarly occur.

If the exercising subject inadvertently or deliberately chooses not to follow the controller commands, a large tracking error will be generated, and the controller will require a huge effort to compensate for the absent effort. This may result in a large fluctuation in the HR of the subjects if the exercising subject responds to the biofeedback signal. If the HR profile (such as warm up slope $\theta_{w}$ in (1) and target $\mathrm{HR}\left(H R_{e}\right)$ ) is not determined accurately for each subject, considering the maximum work-rate that the subject can do whilst pedalling continuously, a collapse in the closed-loop mechanism can occur. Even if the target HR and warm up slope are set appropriately according to each subject's condition, the subject may deliberately choose to stop the exercise for a short period.

In order to address the problem, the HR profile needs to be more dynamic. In other words, where large tracking errors are noted (larger than a specific threshold), the HR profile can be temporarily held constant to prevent large efforts being required of the subjects. We thus revise the HR

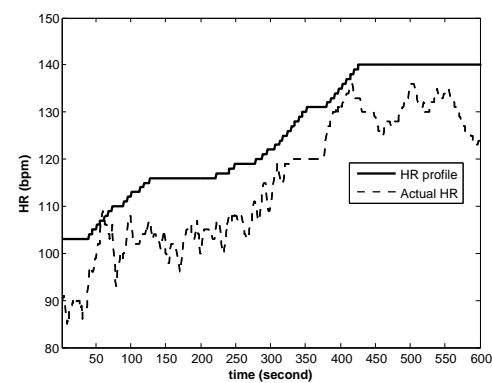

Fig. 2.5 Dynamic profile mechanism used as anti-windup

profile as follows:

$\theta_{w}= \begin{cases}\frac{H R_{e}-H R_{n}}{T_{w}} & \text { if } e(k) \leq \delta, \\ 0 & \text { if } e(k)>\delta,\end{cases}$

where $\delta>0$ denotes a certain threshold. Notice that the warm up duration will then increase to $\bar{T}_{w}=T_{w}+T_{a}$ in which $T_{a}$ is the summation of all time periods that $e(t)>\delta$ and $\theta_{w}=0$. This can avoid any large fluctuation in the HR of the subject. Fig. 2.5 manifests the concept of a dynamic profile during a trial exercise. Here the tracking error threshold is assumed to be $\delta=10$.

On the other hand, since the designed control system contains an auditory converter (biofeedback generator) it is also possible that both converters (audio frequency and time duration) may reach their limitations, even with the accurate definition of the translating functions $t_{d}$ and $f_{b}$ in (6) and (7), respectively. This can also result in windup phenomena. Note that the velocity form PID control strategy first computes $\Delta u(k)$, see (3), which is then integrated via an integrator. This possible windup problem can readily be addressed by assuming $\Delta u(k)=0$ during the saturation. Thus, we define

$$
\begin{cases}u(k)=u(k-1)+\Delta u(k), & \text { if }\left\{\begin{array}{l}
\lambda_{1}<g(u(k))<\lambda_{2} \& \\
\gamma_{1}<h(u(k))<\gamma_{2}
\end{array}\right. \\
u(k)=u(k-1), & \text { otherwise. }\end{cases}
$$

\subsection{Tuning the PID controller gains}

This subsection describes the system identification method that we used to make a model describing a system from the measured input-output test data. Participants in Group 1 and 2 (see Table 1) were requested to complete an exercise session. In this session, the subject was requested to complete an exercise on the cycle-ergometer with the following setup. In order to excite the system dynamics, we sent a minimum possible biofeedback for $10 \mathrm{~s}$ which is equivalent to auditory commands with $t_{d}=500 \mathrm{~ms}$ and $f_{b}=400 \mathrm{~Hz}$ ) to the subjects. During this period the subjects needed to exercise 
with the minimum possible rate. Then we changed the auditory commands to the maximum case, which is equivalent to auditory commands with $t_{d}=60 \mathrm{~ms}$ and $f_{b}=2000 \mathrm{~Hz}$. Using the Matlab identification toolbox, the so-called average transfer function could be obtained. Based on the achieved average transfer function, we optimised PID gains to have a concurrent fast and stable response using the Matlab PID tuner toolbox. This scheme improved the robustness of the PID controller against the inter-individual differences of the HR dynamics, while achieving a good performance as well.

\subsection{Control performance validation}

In order to validate the proposed control system, twenty four healthy male subjects (Group 1 and 2 in Table 1) were asked to exercise on the cycle-ergometer. The PID controller parameters obtained from the average transfer functions of Group 1 and Group 2 were tuned to $K_{p}=1.9040, K_{i}=0.0244$ and $K_{d}=0.0013$, and $K_{p}=2.0030, K_{i}=0.0259$ and $K_{d}=$ 0.0011 , respectively, in which their robustness against the inter-individual differences in the dynamic HR response to work rate was verified, demonstrated in the Result section, using a statistical hypothesis test. To do so, we tested the set of PID parameters obtained from Group 1 on Group 2 and vice versa. The HR profile was defined in 3 stages; warm up, aerobic training and cool down, where the aerobic training stage lasted 5, 5 and 7 minutes for each subject respectively. Moreover, the reference value of $125 \mathrm{bpm}$ was the desired aerobic training HR for all subjects during the exercise stage.

For comparison, twenty four participants were asked to exercise again, using a conventional PID controller with the same gains given above, and further, using a fixed-rate biofeedback mechanism (subjects were provided with an auditory stimulus every $1 s$ using the last updated control signal; see [3]). Except the controller and biofeedback rate, the experiments were implemented with the same setup of the previous experiments.

\subsection{Statistics}

Firstly, a statistical analysis was carried out to ensure the robustness of the the identified PID parameters against the inter-individual differences of the HR dynamics, i.e. whether significant differences existed between performances of the PID controllers tuned by two sets of parameters, using unpaired two-sample t-test. A second statistical hypothesis test was carried out to compare the performance of the proposed actuator-based event-driven PID controller and a conventional control mechanism that uses a fixed-rate biofeedback (using paired two-sided t-test). The significance level was

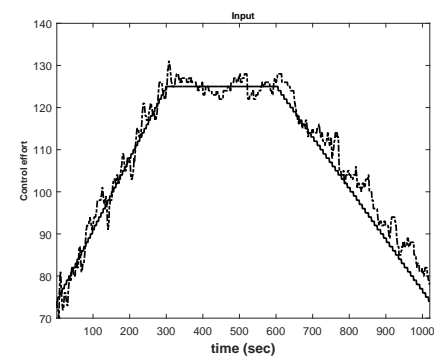

Fig. 3.1 HR profile tracking during cycling using event-driven PID controller

set to $5 \%$, i.e. $p<0.05$, for all analyses. Statistical calculations were performed using the Matlab Statistics Toolbox.

\section{Results}

In order to evaluate the performance of different controllers quantitatively, we used the discrete-time cumulative root mean square (RMS) value of the tracking error, as

$e_{r m s} \triangleq \sqrt{\frac{1}{N} \sum_{i=1}^{N}|e(i)|^{2}}$

where $N$ denotes the number of samples. Indeed, the RMS value gives a measure of the average energy in the error signal. Analysis of all outcomes for all subjects in Group 1 and 2 over the 3 stages HR profile, using the actuatorbased event-driven PID controller, showed that there were no significant differences in the mean values of $e_{r m s}[3.9 \mathrm{vs}$. $3.7 \mathrm{bpm}, p=0.65$ ] between two different sets of PID gains obtained via the average transfer function of Group 1 and Group 2; see Table 2.

The second sub-analysis of difference between the performance of the proposed synchronised-rate event-driven PID controller and fixed-rate conventional PID controller revealed a significant difference in the level of RMS value of the tracking error for Group 1 [3.9 vs. $5.0 \mathrm{bpm}, p<0.05]$ and Group 2 [3.7 vs. 4.4 bpm, $p<0.05$ ]; refer to Table 3. For illustration of the tracking performance of the novel synchronisedrate event-driven PID controller, a single result for a subject (in Group 1) is demonstrated in Fig. 3.1. Moreover, the performance of the fixed-rate controller for this subject can be seen in Fig. 3.2. While the RMS value of the tracking error for this specific subject in Fig. 3.1 (exercising with synchronised-rate biofeedback) is $3.4485 \mathrm{bpm}$, this value is $4.9307 \mathrm{bpm}$ in Fig. 3.2. A box-plot of the distribution of $e_{r m s}$ in Group 1 and 2 using synchronised-rate and fixed-rate control mechanisms is shown in Fig. 3.3. 
"Manuscript-SP-R3" - 2016/7/4 - 12:15 - page 8 - \#8

Table 2 Statistical analysis of the generalisation of the tuned PID parameters

\begin{tabular}{cccc}
\hline \hline & Group 1 with PID tune of Group 2 & Group 2 with PID tune of Group 1 & $p$ value \\
\hline$e_{r m s}$ & $3.9 \pm 0.9,3.7-4.1$ & $3.7 \pm 1.1,3.5-3.9$ & 0.65 \\
\hline
\end{tabular}

Values: mean \pm standard deviation, $95 \%$ confidence interval for the mean value $p$ value is for unpaired two-sample t-test

Degree of freedom $=22$

Table 3 Comparison of synchronised-rate and fixed-rate control mechanism

\begin{tabular}{cccc}
\hline \hline & Proposed event-driven control system $\left(e_{r m s}\right)$ & Conventional fixed-rate control system $\left(e_{r m s}\right)$ & $p$ value \\
\hline Group 1 & $3.9 \pm 0.9,3.7-4.1$ & $5.0 \pm 1.2,4.8-5.3$ & 0.0004 \\
\hline Group 2 & $3.7 \pm 1.1,3.5-3.9$ & $4.7 \pm 1.2,4.5-5.0$ & 0.0001 \\
\hline
\end{tabular}

Values: mean \pm standard deviation, $95 \%$ confidence interval for the mean value $p$ value is for paired two-sided t-test

Degree of freedom $=11$

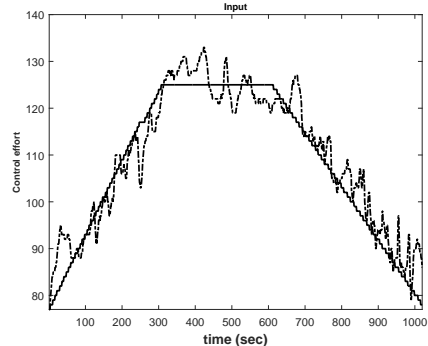

Fig. 3.2 HR profile tracking during cycling using conventional PID controller and fixed-rate biofeedback

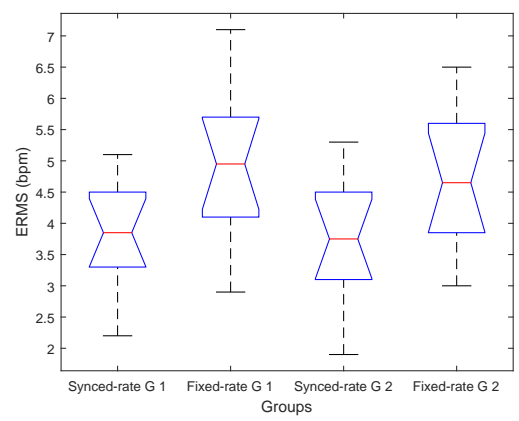

Fig. 3.3 Distribution of $e_{r m s}$ in Group 1 and 2 using synchronised-rate and fixed-rate mechanism

\section{Discussion}

The aim of this study was to develop a novel actuator-based event-driven control mechanism for HR regulation during cycle-ergometer exercise, thereby investigating the improvement of the performance of this controller compared to a conventional fixed-rate control mechanism with respect to RMS value of the tracking error.
As we used a non-model-based PID controller in this study, we needed to set its parameters and further investigate the generalisation of the obtained parameters. With regard to the PID gains, analysis of the outcomes, obtained through cross-validation method, showed no significant differences in RMS value of the tracking error. This observation confirms the proposition of the generalisation of the obtained PID gains. Furthermore, this achieved result is in-line with that of in [13], that is an overall nominal model can statistically be derived which can be employed in the design of feedback controllers for HR regulation applications. Hence, despite the fact that the HR response dynamics vary between individuals, a robust non-model-based LTI feedback controller can be obtained, through a nominal average HR response dynamics, that can neutralise the plant nonlinearities and uncertainties; see [13]. Nevertheless, a different research can focus on time-varying (adaptive) and/or nonlinear control techniques to compare their performances relative to a non-model-based LTI controller.

On the other hand, with regard to the type of biofeedback transmission rate (synchronised vs. fixed), analysis of outcomes obtained separately over Group 1 and 2 (see Table 3) showed significant differences in the mean value of the RMS values of the tracking error. This observation leads to this proposition that the novel synchronised-rate biofeedback mechanism improves tracking performance and reduces the possibility of the subject's cognitive disengagement from the feedback controller compared to the fixed-rate control mechanism. Furthermore, as can be seen in Fig. 3.1, the proposed event-driven control mechanism smoothes out the abrupt variations in the HR profile preventing overshooting in the HR responses, and allows the HR to change in a more gradual manner.

Furthermore, referring to the obtained statistical results (given in Table 3), it is found that the fixed-rate biofeed- 
"Manuscript-SP-R3" — 2016/7/4 - 12:15 — page 9 — \#9

back mechanism (e.g. in [3]) is not a very successful one to instruct the subjects to change their exercise rate according to the PID controller signal, as the rate of biofeedback is not synchronous with the rate of exercise rate (pedalling rate in this case). Referring to the experiences reported by the exercise participants, this method may lead to cognitive disengagement of the exercising subject from the controller signals and, consequently, larger overshooting in the HR responses. This can be a major drawback of the control system, especially in cardiac rehabilitation programs. Specifically, during the warm up and cooling down periods (the first and third stages of the HR profile), as the pedalling rate varies repeatedly, it is hard for the exerciser to follow the fixed-rate biofeedback signal and a confusion may occur. Due to the confusion during the warm up stage, the subject may not successfully follow the control commands and large fluctuations may occur in the HR response during the aerobic training stage (second stage). Indeed, the results from the second sub-analysis lead to the hypothesis that the synchronised-rate event-driven control mechanism presented in this paper makes it possible to more precisely regulate HR to a predetermined HR profile compared to the fixed-rate control mechanism.

It should also be pointed out that the subjects' HR did not completely follow the HR profile during the cooling down period. This is because the recovery HR of the subjects could not be further reduced since the subjects had already stopped their exercise. In other words, if a subject has a high resting HR which is higher than the reference HR, nothing could be done by the control system to reduce the subject's resting HR. However, in such a case, the cooling down period aims to prevent possible venous pooling and reduced venous return to the heart from an excessively abrupt termination to the exercise.

\section{Conclusions}

In this study, an automated system has been designed to help exercising subjects to track a predetermined HR profile. The PID controller, which is simple to design and implement, was deployed and tested in this project. The significance of this study, compared to other published data, is the fact that the biofeedback signals are synchronised with respect to the positions of the pedals. To this end, in the case of the PID controller, by adjusting the time duration and the frequency of the auditory signals, the HR of the subjects is forced to track the profile. To implement this idea, a new control system strategy has been designed, termed an actuator-based event-driven control system. In addition, for comparison, a conventional PID controller using a fixed-rate biofeedback mechanism has been used with its performance compared to the actuator-based event-driven PID control. Statistical analysis which were carried out on twenty four healthy male sub- jects revealed that the proposed synchronised-rate biofeedback mechanism significantly improves the tracking performance of the designed automated exercise testing system compared to the fixed-rate mechanism.

\section{References}

1. Aronow, W.S.: Exercise therapy for older persons with cardiovascular disease. The American journal of geriatric cardiology 10(5), 245-252 (2001)

2. Åström, K.J., Hägglund, T.: Advanced PID control. ISA-The Instrumentation, Systems, and Automation Society; Research Triangle Park, NC 27709 (2006)

3. Baig, D.e.Z., Javed, F., Savkin, A.V., Celler, B.G.: An adaptive $H_{\infty}$ control design for exercise-independent human heart rate regulation system. In: 9th IEEE International Conference on Control and Automation (ICCA), pp. 1033-1036 (2011)

4. Cheng, T.M., Savkin, A.V., Celler, B.G., Su, S.W., Wang, L.: Nonlinear modeling and control of human heart rate response during exercise with various work load intensities. Biomedical Engineering, IEEE Transactions on 55(11), 2499-2508 (2008)

5. Colombo, G., Joerg, M., Schreier, R., Dietz, V., et al.: Treadmill training of paraplegic patients using a robotic orthosis. Journal of rehabilitation research and development 37(6), 693-700 (2000)

6. Cooper, R., Horvath, S., Bedi, J., Drechsler-Parks, D., Williams, R.: Maximal exercise response of paraplegic wheelchair road racers. Spinal Cord 30(8), 573-581 (1992)

7. Diggle, P.J.: Time SeriesA Biostatistical Introduction. Oxford Univ. Press, Oxford, UK (1990)

8. Fletcher, G.F., Balady, G.J., Amsterdam, E.A., Chaitman, B., Eckel, R., Fleg, J., Froelicher, V.F., Leon, A.S., Piña, I.L., Rodney, R., et al.: Exercise standards for testing and training a statement for healthcare professionals from the american heart association. Circulation 104(14), 1694-1740 (2001)

9. Giardino, N.D., Lehrer, P.M., Edelberg, R.: Comparison of finger plethysmograph to ECG in the measurement of heart rate variability. Psychophysiology 39(2), 246-253 (2002)

10. Giggins, O.M., Persson, U., Caulfield, B.: Biofeedback in rehabilitation. J Neuroeng Rehabil 10(1), 60 (2013)

11. Hajek, M., Potuček, J., Brodan, V.: Mathematical model of heart rate regulation during exercise. Automatica 16(2), 191-195 (1980)

12. Heemels, W., Sandee, J., Van Den Bosch, P.: Analysis of eventdriven controllers for linear systems. International journal of control 81(4), 571-590 (2008)

13. Hunt, K.J., Fankhauser, S.E., Saengsuwan, J.: Identification of heart rate dynamics during moderate-to-vigorous treadmill exercise. Biomedical engineering online 14(1), 117 (2015)

14. Kawada, T., Ikeda, Y., Takaki, H., Sugimachi, M., Kawaguchi, O., Shishido, T., Sato, T., Matsuura, W., Miyano, H., Sunagawa, K.: Development of a servo-controller of heart rate using a cycle ergometer. Heart and vessels 14(4), 177-184 (1999)

15. McCrory, M.A., Mole, P.A., Nommsen-Rivers, L.A., Dewey, K.G.: Between-day and within-day variability in the relation between heart rate and oxygen consumption: effect on the estimation of energy expenditure by heart-rate monitoring. The American journal of clinical nutrition 66(1), 18-25 (1997)

16. Mersy, D.: Health benefits of aerobic exercise. Postgraduate medicine 90(1), 103-7 (1991)

17. Montoya, R., Dupui, P., Pages, B., Bessou, P.: Step-length biofeedback device for walk rehabilitation. Medical \& Biological Engineering \& Computing 32(4), 416-420 (1994)

18. Paradiso, M., Pietrosanti, S., Scalzi, S., Tomei, P., Verrelli, C.M. Experimental heart rate regulation in cycle-ergometer exercises. IEEE Transactions on Biomedical Engineering 60(1), 135-139 (2013) 
19. Petrofsky, J.: New algorithm to control a cycle ergometer using electrical stimulation. Medical \& Biological Engineering \& Computing 41(1), 18-27 (2003)

20. Robergs, R.A., Landwehr, R.: The surprising history of the "hrmax= 220-age" equation. J Exerc Physiol 5(2), 1-10 (2002)

21. Su, S.W., Huang, S., Wang, L., Celler, B.G., Savkin, A.V., Guo, Y., Cheng, T.: Nonparametric Hammerstein model based model predictive control for heart rate regulation. In: IEEE-EMBS, pp. 2984-2987 (2007)

22. Su, S.W., Huang, S., Wang, L., Celler, B.G., Savkin, A.V., Guo, Y., Cheng, T.M.: Optimizing heart rate regulation for safe exercise. Annals of biomedical engineering 38(3), 758-768 (2010)

23. Su, S.W., Wang, L., Celler, B.G., Savkin, A.: Heart rate control during treadmill exercise. In: IEEE-EMBS, pp. 2471-2474 (2006)

24. Su, S.W., Wang, L., Celler, B.G., Savkin, A.V., Guo, Y.: Identification and control for heart rate regulation during treadmill exercise. IEEE Transactions on Biomedical Engineering 54(7), 1238-1246 (2007)

25. Yoshino, K., Adachi, K., Ihochi, K., Matsuoka, K.: Modeling effects of age and sex on cardiovascular variability responses to aerobic ergometer exercise. Medical \& biological engineering \& computing 45(11), 1085-1093 (2007)
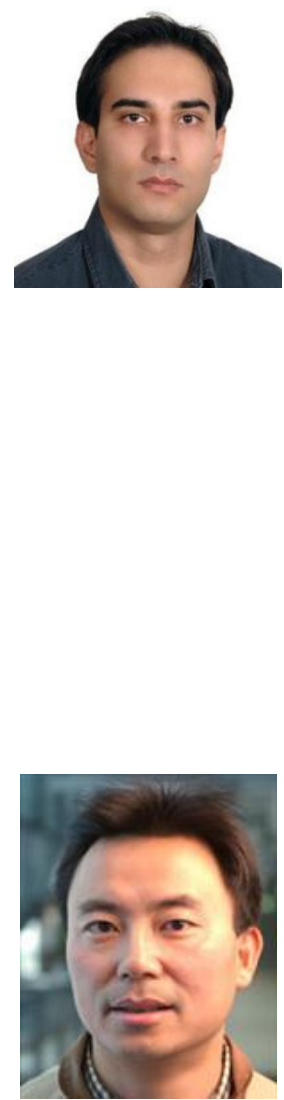

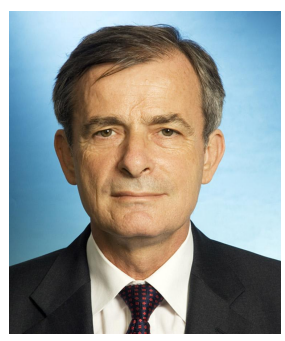

Branko G. Celler received B.Sc. and B.E. (Hons.) degrees in Electrical Engineering and a Ph.D. degree in Biomedical Engineering from the UNSW, Australia. He is currently a Research Professor at the University of NEW South Wales School of Electrical Engineering. His research interests include biomedical instrumentation and systems, noninvasive modeling of cardiovascular performance, and medical informatics.

\begin{abstract}
Ahmadreza Argha received B.S. and M.S. degrees in Electrical Engineering from Shiraz University, Iran. He is currently a Ph.D. student at UTS in Sydney, Australia. His research interests include biomedical system modelling and control, robust control, and network systems.
\end{abstract}

Steven W. Su received B.S. and M.S. degrees from Harbin Institute of Technology (HIT), China, and a Ph.D. degree from the ANU, Australia. He is currently an Associate Professor at UTS. His research interests include biomedical system modelling and control, robust and adaptive control. 